\title{
Tumor necrosis factor $\alpha$ knockout impaired tumorigenesis in chronic myeloid leukemia cells partly by metabolism modification and miRNA regulation
}

This article was published in the following Dove Medical Press journal:

OncoTargets and Therapy

Na Shen, ${ }^{1, *}$ Songya Liu, ${ }^{2, *}$ Jieke Cui, ${ }^{3}$ Qing Li, ${ }^{4}$ Yong You, ${ }^{1}$ Zhaodong Zhong,' Fanjun Cheng,' An-Yuan Guo, ${ }^{5}$ Ping Zou,' Guolin Yuan, ${ }^{6}$ Xiaojian Zhu ${ }^{2}$

'Institute of Hematology, Union Hospital, Tongji Medical College, Huazhong University of Science and Technology, Wuhan 430022, China; ${ }^{2}$ Department of Hematology, Tongji Hospital, Tongji Medical College, Huazhong University of Science and Technology, Wuhan 430030, China; ${ }^{3}$ Department of Hematology, The First Affiliated Hospital of Zhengzhou University, Zhengzhou 450052, China; ${ }^{4}$ Department of Hematology, Wuhan No I Hospital, Wuhan 430022, China; ${ }^{5}$ Key Laboratory of Molecular Biophysics of the Ministry of Education, College of Life Science and Technology, Huazhong University of Science and Technology, Wuhan 430074, China; ${ }^{6}$ Department of Hematology, Xiangyang Central Hospital, The Affiliated Hospital of Hubei University of Arts and Science, Xiangyang 441000, China

*These authors contributed equally to this work
Correspondence: Xiaojian Zhu

Department of Hematology, Tongji Hospital,

Tongji Medical College, Huazhong University of Science and Technology, No 1095 Jiefang Road, Wuhan 430030, China

Tel +86 I507 I I I I I92

Fax +86 2783662680

Email zhuxiaojian@hust.edu.cn

Guolin Yuan

Department of Hematology, Xiangyang Central Hospital, The Affiliated Hospital of Hubei University of Arts and Science, No 136 Jingzhou Street, Xiangyang 441000, China Tel +86 18696231603 Email yuanguolin1973@sina.com
Purpose: Half of the chronic myeloid leukemia (CML) patients with sustained deep molecular response suffer from relapse after discontinuation mainly because tyrosine kinase inhibitors (TKIs) cannot eradicate leukemia stem cells (LSCs). In addition, tumor necrosis factor $\alpha$ (TNF- $\alpha$ ) is highly detected in CML patients. Our aim was to explore whether TNF- $\alpha$ is a potential target for LSC elimination.

Materials and methods: We applied a CRISPR/Cas9 gene editing technique, colonyforming cell assay, subcutaneous tumor models, miRNA-seq and liquid chromatography-mass spectroscopy (LC-MS) on metabonomics to explore the feasibility and mechanism of TNF- $\alpha$ as a new therapeutic target for CML.

Results: We demonstrated that TNF- $\alpha$ knockout remarkably decreased the proliferative, colony-forming and in vivo tumorigenesis capacities of the CML K562 cell line. The apoptosis was increased when TNF- $\alpha$ knockout cells were cultured with imatinib. The mechanisms involved in the abovementioned phenomena were that TNF- $\alpha$ knockout inhibited the citrate cycle and increased starch, sucrose, amino sugar and nucleotide sugar metabolism. In addition, differentially expressed miRNAs between TNF- $\alpha$ knockout and control cells were involved in the cell cycle, CML, P13K-Akt and pathways in cancer.

Conclusion: We identified that TNF- $\alpha$ may serve as a new target therapy for CML and described the metabolic pathways associated with TNF- $\alpha$ in CML cells for the first time.

Keywords: chronic myeloid leukemia, tumor necrosis factor $\alpha$, CRISPR/Cas9 gene editing, tumorigenesis, metabolism profile

\section{Introduction}

Chronic myeloid leukemia (CML) is a myeloproliferative disease caused by the translocation and fusion of the $A B L$ gene on chromosome 9 and the $B C R$ gene on chromosome 22, which encodes the BCR-ABL1 oncoprotein, a constitutively active tyrosine kinase enzyme. ${ }^{1,2}$ Tyrosine kinase inhibitors (TKIs) have revolutionized CML therapy, with 8 -year overall survival probability above $80 \%{ }^{3}$ However, TKIs can effectively target proliferating mature cells, but they do not eradicate quiescent leukemia stem cells (LSCs), for which cell survival is independent of BCR-ABL1, ${ }^{4}$ resulting in relapse in $40 \%-60 \%$ of the patients after discontinuation..$^{5-7}$ In addition, there is a potential risk of TKI resistance due to mutations in $B C R-A B L 1$ and disease progression. ${ }^{8}$ Therefore, recognizing new therapeutic targets or combining TKIs to eradicate CML LSCs could be a new strategy to cure CML. 
Tumor necrosis factor $\alpha$ (TNF- $\alpha$ ), a pleiotropic cytokine exerting both inhibitory and stimulatory effects on diverse cellular processes, is a key regulator of immunological responses. ${ }^{9}$ It has been reported that CML stem/progenitor cells (CML-SPCs) produce TNF- $\alpha$ at higher levels in a kinase-independent fashion. Inhibition of autocrine TNF- $\alpha$ signaling induces apoptosis, an effect that is more significant when combined with nilotinib. ${ }^{10}$ In acute myeloid leukemia (AML), leukemia-initiating cells (LICs) can perform autocrine TNF- $\alpha$ signaling, which forms an NF- $\mathrm{KB} / \mathrm{TNF}-\alpha$ feedback loop to promote the survival of LICs. ${ }^{11}$ In addition, TNF restricts normal hematopoietic stem cell (HSC) activity depending on the expression of TNF- $\alpha$ receptor 1 (TNFR1) and TNFR2. ${ }^{12}$ Targeting cell surface antigens that exhibit druggable features and play critical roles in leukemia maintenance represents an attractive strategy. Clinical trials with TNF- $\alpha$ antagonist have shown promising clinical activity against solid tumors such as pancreatic tumor, renal cell carcinoma and metastatic breast cancer. ${ }^{11,13}$

Increasing evidence has shown that specific modes of metabolism play important roles in the self-renewal capacities of both healthy and transformed stem cells. ${ }^{14,15}$ LSCs demand tightly regulated metabolism since the disruption of either glycolysis or mitochondrial respiration impairs leukemogenesis. ${ }^{16,17}$ This is the first study to adopt a CRISPR/Cas9-mediated TNF- $\alpha$ knockout K562 model to investigate the potential metabolism mechanisms by which TNF- $\alpha$ is involved in CML.

\section{Materials and methods}

\section{Cell culture and drug treatment}

The human CML cell line K562 was purchased from the China Center for Type Culture Collection (Wuhan, China) and cultured in RPMI (Hyclone, Logan, UT, USA) supplemented with 10\% FBS (NQBB, Adelaide, Australia). The T293 cell line was cultured in DMEM (Thermo Fisher Scientific, Waltham, MA, USA) supplemented with $10 \%$ FBS in an incubator at $37^{\circ} \mathrm{C}$ with $5 \% \mathrm{CO}_{2}$. Cells were passaged with pancreatin (Merck, St Louis, MO, USA) when approximately $70 \%-90 \%$ confluence was reached. Imatinib was kindly supplied by Novartis International AG (Basel, Switzerland).

\section{CRISPR/Cas9 gene editing}

The 2 sgRNAs targeting exon 1 of TNF- $\alpha$ were designed using an online application (http://tools.genome-engineering.org). Next, 2 sgRNAs were synthesized and ligated to the U6-sgRNA cloning vector to form TNF- $\alpha$ sgRNA-expressing plasmids. K562 cells $\left(1-2 \times 10^{6}\right)$ were resuspended in transfection medium (containing $82 \mathrm{~mL} \mathrm{SF}$ medium and $18 \mathrm{~mL}$ supplement medium), and then $2 \mathrm{mg}$ of each TNF- $\alpha$
sgRNA plasmid was added. The cells were cultured in an incubator at $37^{\circ} \mathrm{C}$ with $5 \% \mathrm{CO}_{2}$ for $48-72 \mathrm{~h}$ after electroporation, and then the fluorescence was observed. We adopted flow cytometry to select monoclones for single cell culture and expansion. The cell colonies were subcultured and a part of each clone was collected for PCR amplification and sequencing to screen clones with TNF- $\alpha$ knockout.

\section{Quantitative reverse transcription PCR (qRT-PCR)}

Total RNA was extracted using Trizol reagent. cDNA was synthesized by the reverse transcription of $500 \mathrm{ng}$ of total RNA with the PrimeScript ${ }^{\mathrm{TM}}$ RT Reagent Kit (Takara, Dalian, China). Real-time PCR was performed with $5 \mu \mathrm{L}$ SYBR Premix Ex Taq (Takara, Dalian, China), $0.8 \mu \mathrm{L}$ primers, $0.2 \mu \mathrm{L}$ ROX Reference Dye, $3 \mu \mathrm{L}$ RNase-free $\mathrm{H}_{2} \mathrm{O}$ and $1 \mu \mathrm{L}$ cDNA as a template for a final reaction volume of $10 \mu \mathrm{L}$. The PCR cycling conditions were as follows: initial melting at $95^{\circ} \mathrm{C}$ for $30 \mathrm{~s}$ followed by 40 cycles at $95^{\circ} \mathrm{C}$ for $5 \mathrm{~s}$ and $60^{\circ} \mathrm{C}$ for $30 \mathrm{~s}$. Fluorescence intensity was measured using the ABI StepOneplus ${ }^{\mathrm{TM}}$ Real Time PCR System (Waltham, MA, USA). Analysis of the melting curves for the primers was done to confirm the specificity of the PCR products, and the $\mathrm{Ct}$ values for triplicate reactions were averaged. The fold changes in mRNA expression were calculated through relative quantification $\left(2^{-\Delta C \mathrm{Ct}}\right)$.

\section{Western blot}

Membrane proteins were extracted by dedicated kit (Beyotime Biotechnology, Shanghai, China). In each well, $40 \mu \mathrm{g}$ membrane protein samples were separated by SDS-PAGE at $60 \mathrm{~V}$ $30 \mathrm{~min}$ followed by $100 \mathrm{~V}$ for $1.5 \mathrm{~h}$, and then the proteins were transferred to nitrocellulose membranes. The membranes were blocked with $5 \%$ nonfat milk and incubated overnight with monoclonal antibody $(\mathrm{mAb})$ at $4{ }^{\circ} \mathrm{C}$. Blots were developed using a horseradish peroxidase-conjugated rabbit antihuman secondary antibody and a chemiluminescent detection kit (Google Biotech, Wuhan, China).

\section{Colony-forming cell assay}

We mixed $1.6 \mathrm{~g}$ methylcellulose, $50 \mathrm{~mL}$ ultrapure water and $50 \mathrm{~mL} 2 \times$ PRMI 1640 (HyClone) to prepare methylcellulosebased medium and then added $20 \%$ FBS and $1 \%$ antibiotics (100 U/mL of streptomycin and $100 \mathrm{mg} / \mathrm{mL}$ of penicillin) before experimentation. Three hundred cells were suspended in $1 \mathrm{~mL}$ complete methylcellulose-based medium in 24-well plates. Surrounding wells were filled with $1 \mathrm{~mL}$ PBS to reduce evaporation. Clones were scored after 14 days of incubation at $37^{\circ} \mathrm{C}$ with $5 \% \mathrm{CO}_{2}$. 


\section{Apoptosis detection}

Cells were harvested by centrifugation and washed twice with ice-cold PBS. Following this, the cells were stained with Annexin- $\mathrm{V}$-fluorescein isothiocyanate/propidium iodide (FITC/ PI, BD Pharmingen, Franklin Lake, NJ, USA) for $15 \mathrm{~min}$ at room temperature according to the manufacturer's instructions and analyzed via flow cytometry. The results were analyzed on FlowJo V10 (Stanford University, San Francisco, CA, USA).

\section{Cell proliferation assay}

Cell proliferation was measured through cell counting kit-8 (CCK-8; Biosharp, Hefei, China). Three thousand cells resuspended with $200 \mu \mathrm{L}$ medium were plated in triplicate in 96-well plates and incubated for 24, 48 and $72 \mathrm{~h}$. Twenty microliters of CCK-8 reagent was added to the plates, followed by incubation for $4 \mathrm{~h}$ at $37^{\circ} \mathrm{C}, 5 \% \mathrm{CO}_{2}$ incubator. The absorbance at $450 \mathrm{~nm}$ was measured using a multifunctional enzyme marking instrument.

\section{Subcutaneous tumor models}

Animal experiments were approved by the Hubei Province ethics committee and the institutional review board of Tongji Medical College. All mice used received humane care in accordance with the Guide for the Care and Use of Laboratory Animals (National Academies Press, 2011). Mice were randomly divided into 4 groups as follows: K562, 3C9, 1H7 and 1D9. Cells were collected and subcutaneously injected into 3-4-week-old immunodeficient BALB/c nude mice that were intraperitoneally pretreated with $100 \mathrm{mg} / \mathrm{kg}$ cyclophosphamide. The mental states, weights and tumor volumes of mice were periodically monitored. Twenty-one days after subcutaneous injection, the mice were sacrificed.

\section{Liquid chromatography mass spectroscopy (LC-MS) on metabonomics} $\mathrm{K} 562,3 \mathrm{C} 9$ and $1 \mathrm{H} 7$ cell pellets were collected in Eppendorf tube, and then $1000 \mu \mathrm{L}$ of extraction liquid (acetonitrilemethanol-water, 2:2:1, containing internal standard) was added. The samples were vortexed for $30 \mathrm{~s}$, homogenized at $45 \mathrm{~Hz}$ for $4 \mathrm{~min}$, and sonicated for $5 \mathrm{~min}$ in ice-water bath. The homogenate and sonicate cycle was repeated for 3 times, followed by incubation at $-20^{\circ} \mathrm{C}$ for $1 \mathrm{~h}$ and centrifugation at $12,000 \mathrm{rpm}$ and $4^{\circ} \mathrm{C}$ for $15 \mathrm{~min}$. The resulting supernatants were transferred to LC-MS vials and stored at $-80^{\circ} \mathrm{C}$ until the LC-MS analyses using an Ultra High Performance Liquid Chromatography system (1290; Agilent Technologies, Santa Clara, CA, USA). Mass spectroscopy raw data files were converted to the mzML format using ProteoWizard (Palo Alto, CA, USA) and processed by R package XCMS (version 3.2, La Jolla, CA, USA). The preprocessing results generated a data matrix that consisted of the retention time (RT), mass-to-charge ratio $(\mathrm{m} / \mathrm{z})$ values and peak intensity. OSI-SMMS (version 1.0; Dalian ChemDataSolution Information Technology Co. Ltd. Dalian, China) was used for peak annotation after XCMS data processing with in-house MS/MS database. The screening criteria of differently expressed metabolites was variable importance on the projection (VIP) $>1$ and adjusted $p<0.05$. The Kyoto Encyclopedia of Genes and Genomes (http://www.genome. jp/kegg/) and MetaboAnalyst 3.0 (http://www.metaboanalyst.ca/) were utilized to analyze the metabolic pathways.

\section{RNA sequencing and analysis}

We performed small RNA sequencing of $3 \mathrm{C} 9,1 \mathrm{H} 7$ and $\mathrm{K} 562$ cells with HiSeq2500 at WuXi Genome Center, China. After filtering low-quality reads and adaptor trimming, clean reads of the sequencing data were prepared for mapping. Clean reads of small RNA sequencing were mapped to pre-miRNA sequences of homo sapiens from miRBase V21, and the expression level of miRNA was normalized by expressed reads per million (RPM). Additionally, we calculated the differentially expressed genes and miRNAs by cutoff $\mid \log _{2}($ fold change $) \mid>1$, FPKM $>10$ or RPM $>100$. miRNA target analysis was based on TargetScan. Transcription factor target analysis was based on TRANSFAC. Pathway analysis was based on KEGG. The network was drawn by Cytoscape.

The small RNA-seq data are available at NCBI's Sequence Read Archive with the accession number PRJNA505896.

\section{Cell cycle analysis}

K562 cells (both $3 \times 10^{5}$ ) were fixed with $70 \%$ absolute ethyl alcohol overnight. Then, the cells were washed and stained with a mixture of PI and RNAase (BD Pharmingen) at room temperature for $15 \mathrm{~min}$ and analyzed via flow cytometry. The results were analyzed using ModFit LT software (Verity Software House, ME , USA).

\section{Statistical analysis}

Unless otherwise stated, the results are presented as mean \pm SD. Data were analyzed using GraphPad Prism 5.01. One-way ANOVA tests, 2-way ANOVA tests and paired $t$-tests were used to compare groups. $p<0.05$ was accepted as being statistically significant.

\section{Results CRISPR/Cas9-mediated knockout of TNF- $\alpha$ in $\mathrm{K} 562$ cells}

We designed 3 CRISPR/Cas9 genome editing tools (sgRNA1, sgRNA2 and sgRNA3) to establish TNF- $\alpha$ 
knockout in the K562 cell line (Figure 1A shows sgRNA2). The 3 pSpCas9-gRNA plasmids were transfected into K562 cells. A T7E1 mismatch sensitivity assay was applied to assess the cutting effectiveness of pSpCas9-gRNA plasmids in K562 cells. The result showed that exon 1 ( $\sim 600 \mathrm{bp})$ was divided into 2 sections, which were 408 bp and 203 bp (Figure 1B). sgRNA2, which possessed the highest cutting efficiency, was selected as the genome editing tool for followup experiments. Next, sgRNA2 plasmids were transfected into K562, and the transfection efficiency was determined by fluorescence microscopy. Successfully transfected cells were seeded at a low density in 96-well plates for recovery and homogeneous expansion through fluorescence-activated cell sorting. Two weeks later, 192 K562 clones were selected to identify indels characteristic of nonhomologous end joining (NHEJ) by Sanger sequencing. Among a total of 192 K562 clones, 31 clones $(-16.1 \%)$ contained a gene mutation in exon 1 including 21 homozygous deletion clones and 10 heterozygous deletion clones. (Figure $1 \mathrm{C}$ shows a representative wild-type control, named $3 \mathrm{C} 9$, and 2 mutated clones, named 1H7 and 1D9.) No mutation was present at the off-target locus predicted on the CRISPR/Cas9 design website. TNF- $\alpha$ mRNA and protein levels of 1H7 and 1D9 were significantly decreased compared to 3C9 and the parental K562 cell population ( $p<0.05$; Figure 1D and E). The transfection and cutting processes had no impact on the expression of BCR-ABL1 ( $p>0.05$; Figure 1F).

\section{TNF- $\alpha$ knockout significantly impaired the proliferation and colony-forming capacities of $\mathrm{K} 562$ cells}

Whether grown in normal medium or starvation conditions, there were no differences in apoptosis rate among groups at different time points $(24,48$ and $72 \mathrm{~h})$. The apoptosis rates of

A

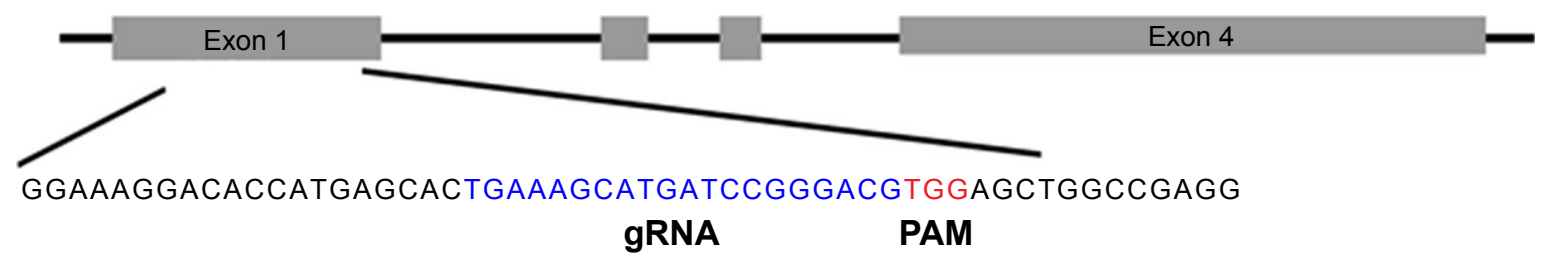

B
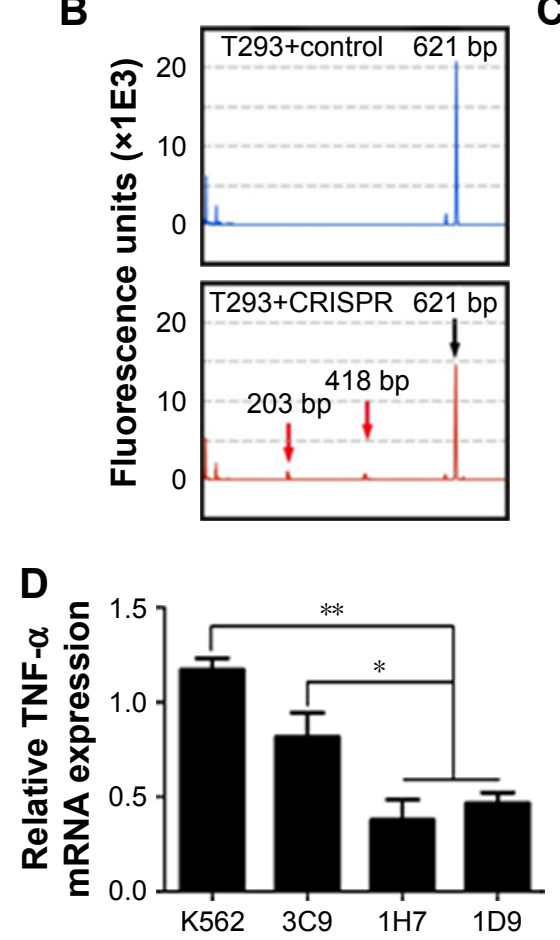

C

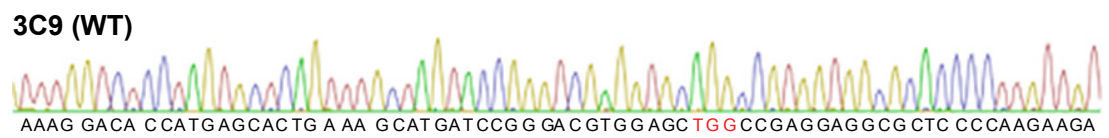

AAAG GACA CCATGAGCACTG AAA GCAT GATCCG GA GTGGAGCTGGCCGAGGAGGGG CTC CCCAAGAAGA $1 \mathrm{H} 7$

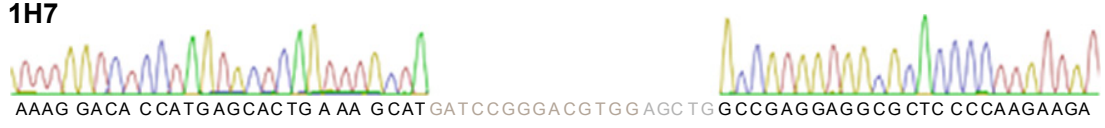
$1 D 9$

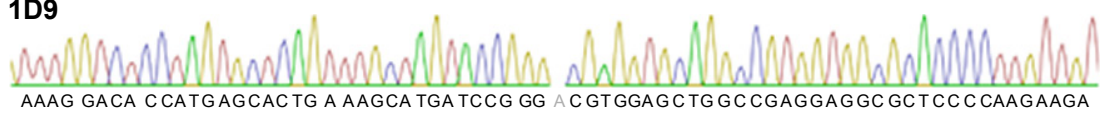

$\mathbf{E}$

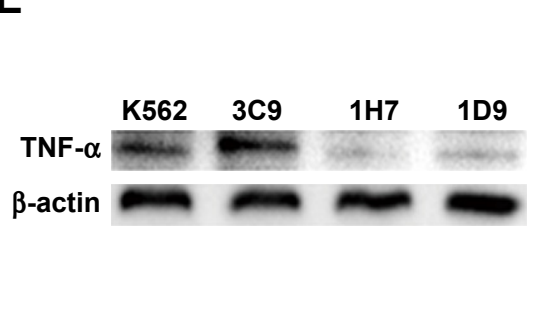

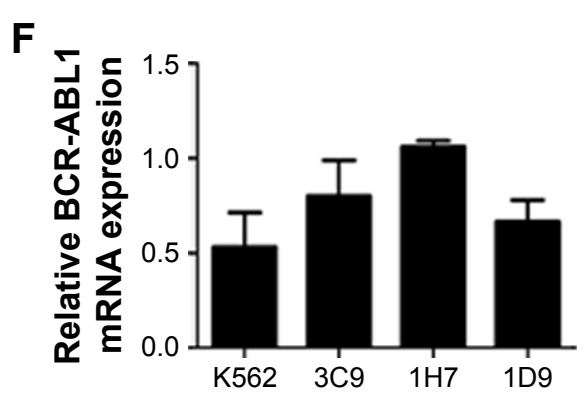

Figure I CRISPR-mediated, targeted knockout of TNF- $\alpha$.

Notes: (A) CRISPR/Cas9 was designed for targeting exon I of TNF- $\alpha$. The blue-colored bases comprise the gRNA targeting sequence, and the red-colored bases are the protospacer adjacent motif. (B) A T7EI mismatch sensitive assay assessed the efficiency of CRISPR/Cas9. (C) Sanger sequencing results of the wild-type clone (3C9) and CRISPR/Cas9-targeted mutant clones (IH7 and ID9) derived from the $\mathrm{K} 562$ cell line. The gray-colored bases denote the $19 \mathrm{bP}$ deletion in IH7 and the I bP deletion in ID9. (D and E) qRT-PCR and Western blot assay confirmed the TNF- $\alpha$ expression levels in K562 cells, wild-type control cells and TNF- $\alpha$ knockout cells. (F) Relative BCR-ABLI mRNA expression in K562 cells, wild-type control cells and TNF- $\alpha$ knockout cells. Error bars represent mean \pm SD of 3 independent experiments. $* p<0.05$ and $* * p<0.0$ I. Abbreviation: qRT-PCR, quantitative reverse transcription PCR. 
starvation groups were higher than those of normal culture groups on the whole (Figure 2A and B). However, mutated clones showed higher rates of apoptosis than wild-type cells when incubated with $5 \mathrm{mM}$ imatinib for $24 \mathrm{~h}$ (Figure 2C). The discrepancies in apoptosis rates were not significant when imatinib was added to each group for 48 or $72 \mathrm{~h}$ (Figure 2C). The proliferation capacities of mutant clones were consistently reduced compared to the corresponding wild-type cells, and this phenomenon was more obvious over time (Figure 2D). In addition, the proliferation of mutant cells was more strongly inhibited when cultured with a low concentration of imatinib (50 nM, Figure 2E). The colonyforming capacities of the mutant clones were decreased compared to wild-type clones and parental cells, and there were no differences between wild-type and parental groups or between 1H7 and 1D9 groups (Figure 2F and G).

\section{TNF- $\alpha$ knockout suppressed the survival of $\mathrm{K} 562$ in vivo}

To further confirm that TNF- $\alpha$ knockout impaired the colonyforming capacity, we conducted in vivo experiments of K562 subcutaneous tumorigenesis in BALB/c nude mice. Tumor sizes were recorded every 3 days. The mice were killed, and their tumors were obtained 21 days after subcutaneous engraftment (Figure 3A). Compared with the control and parental groups, TNF- $\alpha$ knockout effectively reduced the volumes and weights of tumors implanted in nude mice (Figure $3 \mathrm{~B}$ and $\mathrm{C}$ ). There were no differences between
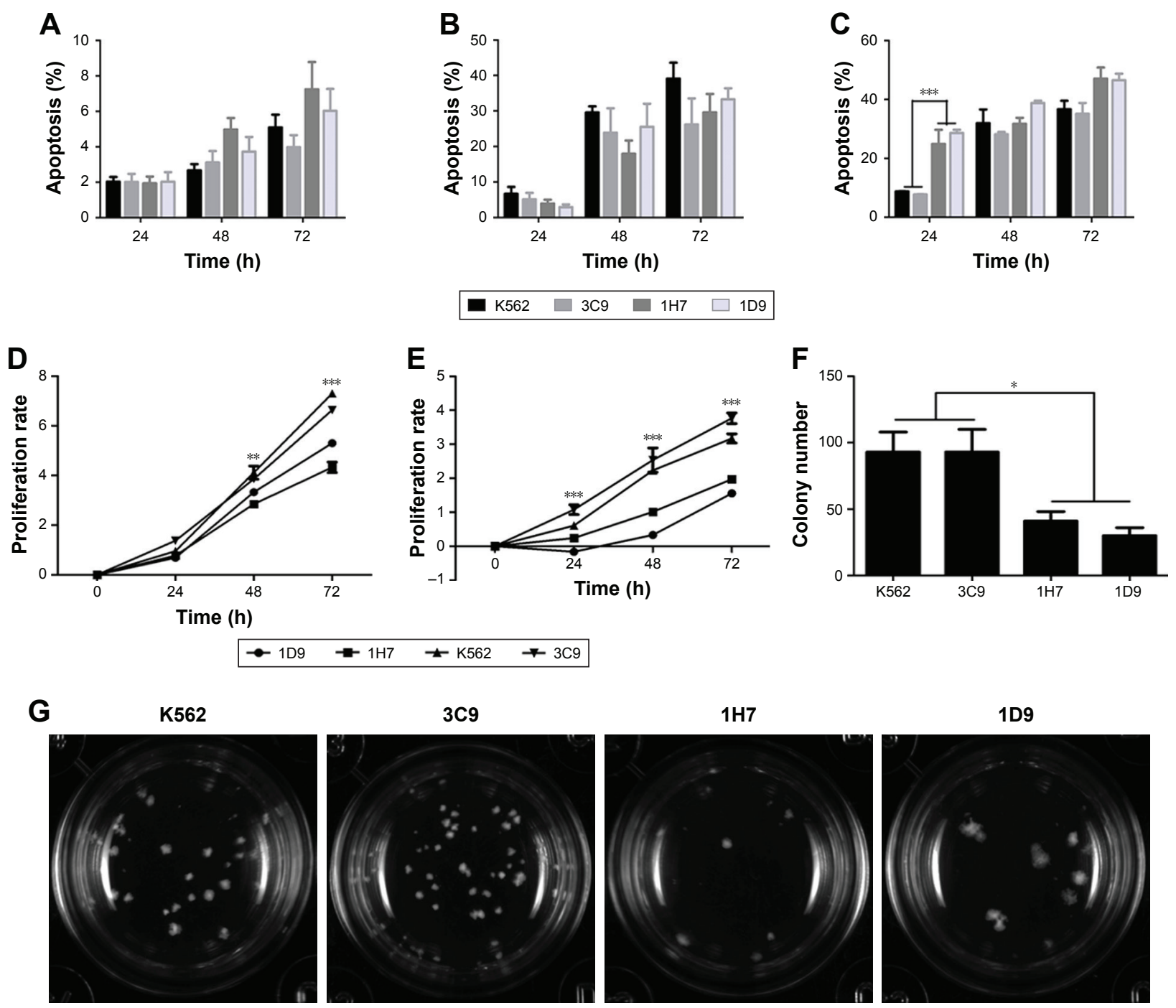

Figure 2 TNF- $\alpha$ knockout impaired the proliferation and colony-forming capacity of K562 cells.

Notes: Apoptosis rates of cells in different culture conditions and at different time points were analyzed by flow cytometry: (A) routine culture, (B) starvation culture and (C) cells incubated with $5 \mu \mathrm{M}$ imatinib. (D and E) Proliferation assay of cells cultured in normal medium or with $50 \mathrm{nM}$ imatinib for $72 \mathrm{~h}$ was analyzed by CCK8 assay. (G) A colony-forming assay was conducted in methylcellulose complete medium. Representative colony images of parental K562 cells (K562), wild-type control cells (3C9) and TNF- $\alpha$ knockout cells (IH7 and ID9). (F) The corresponding statistics for colony numbers for 3 experiments in K562, 3 C9, IH7 and ID9 cells. Error bars represent mean \pm SD of 3 independent experiments. ${ }^{*} p<0.05$, $* * p<0.01$ and $* * * p<0.001$.

Abbreviation: CCK-8, cell counting kit-8. 
A
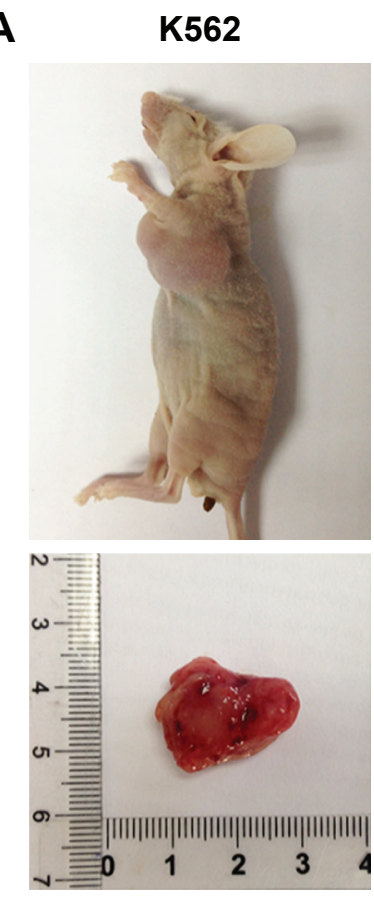

B

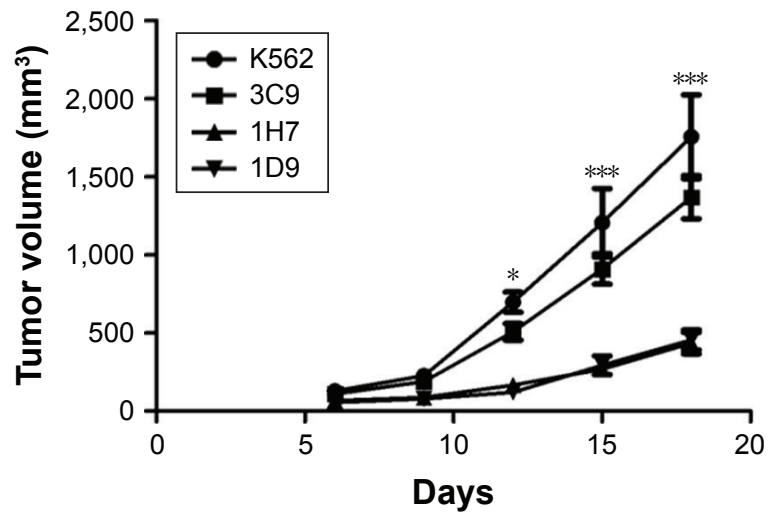

3C9
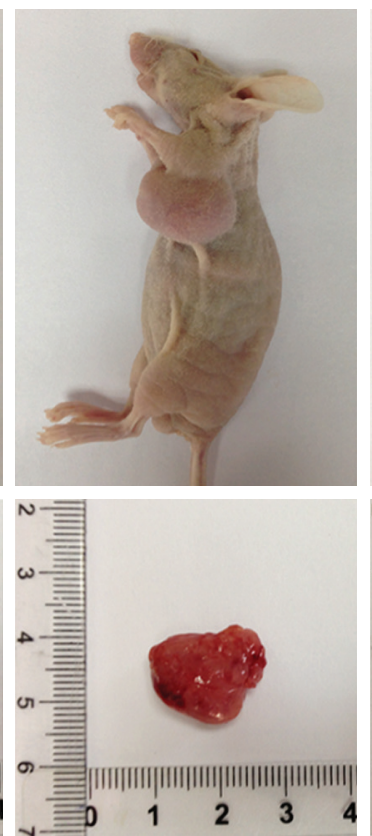

(3)
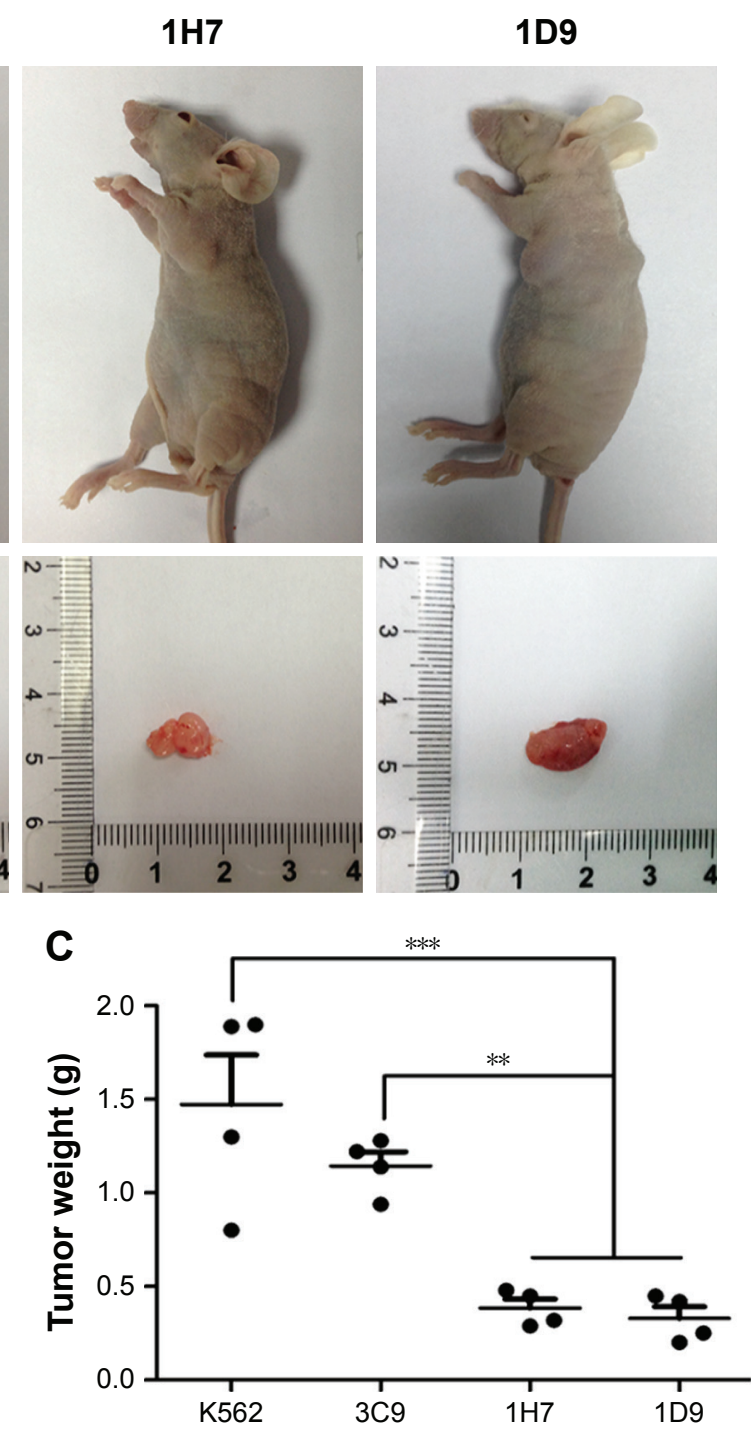

Figure 3 TNF- $\alpha$ knockout weakened the tumorigenesis of $\mathrm{K} 562$ in vivo.

Notes: (A) Representative mice and corresponding tumors 3 weeks after subcutaneous injection of K562, 3C9, IH7 and ID9 cells. (B) The statistics of tumor volume at different times after subcutaneous injection are shown $(n=4)$. (C) The tumor weights of 4 mice per group were analyzed. Error bars represent mean \pm SEM. $* p<0.05$, $* * p<0.01$ and $* * * p<0.001$.

control and parental groups or between 1H7 and 1D9 groups (Figure 3B and C).

\section{TNF- $\alpha$ knockout altered the metabolism profile in $\mathrm{K} 562$ cells}

To explore the potential mechanism of tumor suppression by TNF- $\alpha$ knockout, we employed ultra-performance liquid chromatography mass spectrometry (UPLC-MS)-based metabolomics on K562, 3C9 and 1H7 cells. Orthogonal projections to latent structures-discriminant analysis (OPLSDA) and OPLS-DA permutation tests were used for raw data analysis to obtain reliable metabolite differences between groups (Figure 4A). Hierarchical clustering analysis revealed that uridine diphosphate glucuronic acid and D-glucose 6-phosphate were significantly increased in 3C9 cells; ADP and cis-aconitic acid were notably increased in $1 \mathrm{H} 7$ cells (Figure 4B). These differential metabolites were enriched in starch, sucrose, amino sugar and nucleotide sugar metabolism, citrate cycle and purine metabolism (Figure 4C). The citrate cycle was active in $1 \mathrm{H} 7$ cells; starch, sucrose, amino sugar and nucleotide sugar metabolism were active in 3C9 cells.

\section{TNF- $\alpha$ knockout affected the miRNA regulation}

To investigate how TNF- $\alpha$ affected the phenotype of K562 cells, we also conducted small RNA-seq on K562, $1 \mathrm{H} 7$ and 3C9 cells. The differentially expressed miRNAs were miR-504-5p, miR-23b-3p, miR-3182, miR-1275 and 
A

Intercepts: $R^{2} Y(c u m)=(0,0.99), Q^{2}($ cum $)=(0,-0.04)$

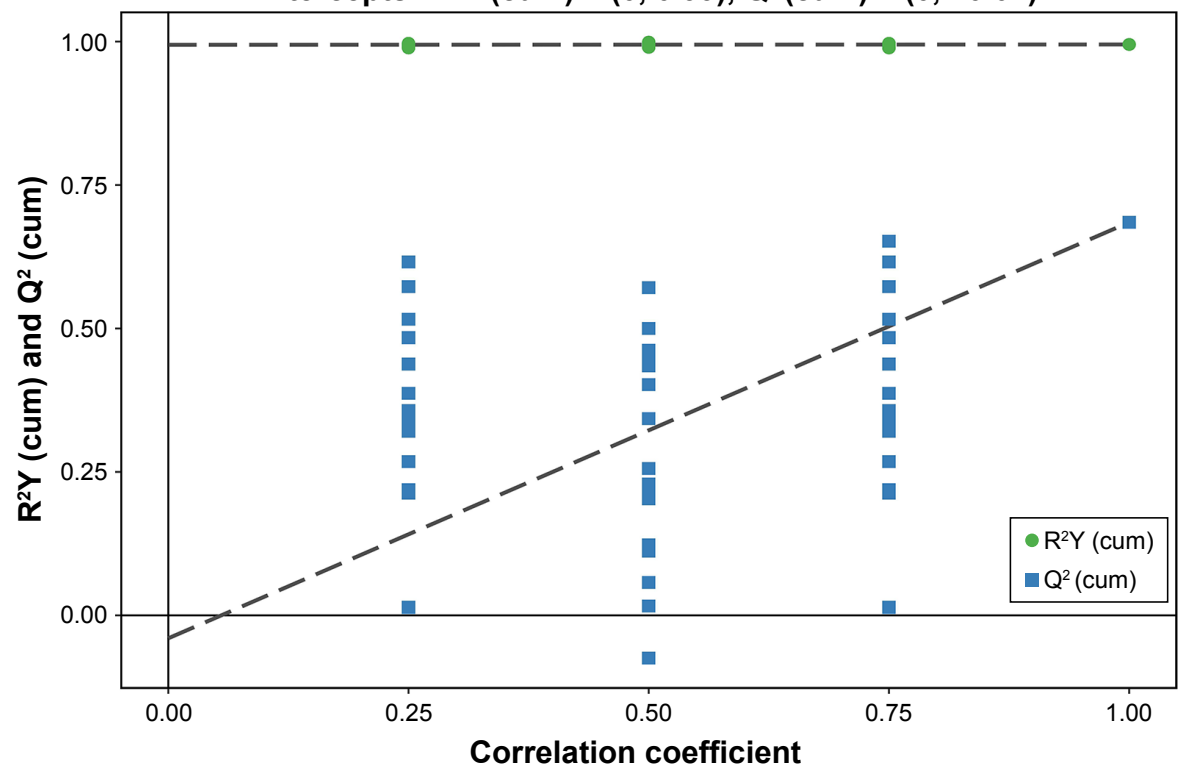

B

$1 \mathrm{H} 7 \quad 3 \mathrm{C} 9$
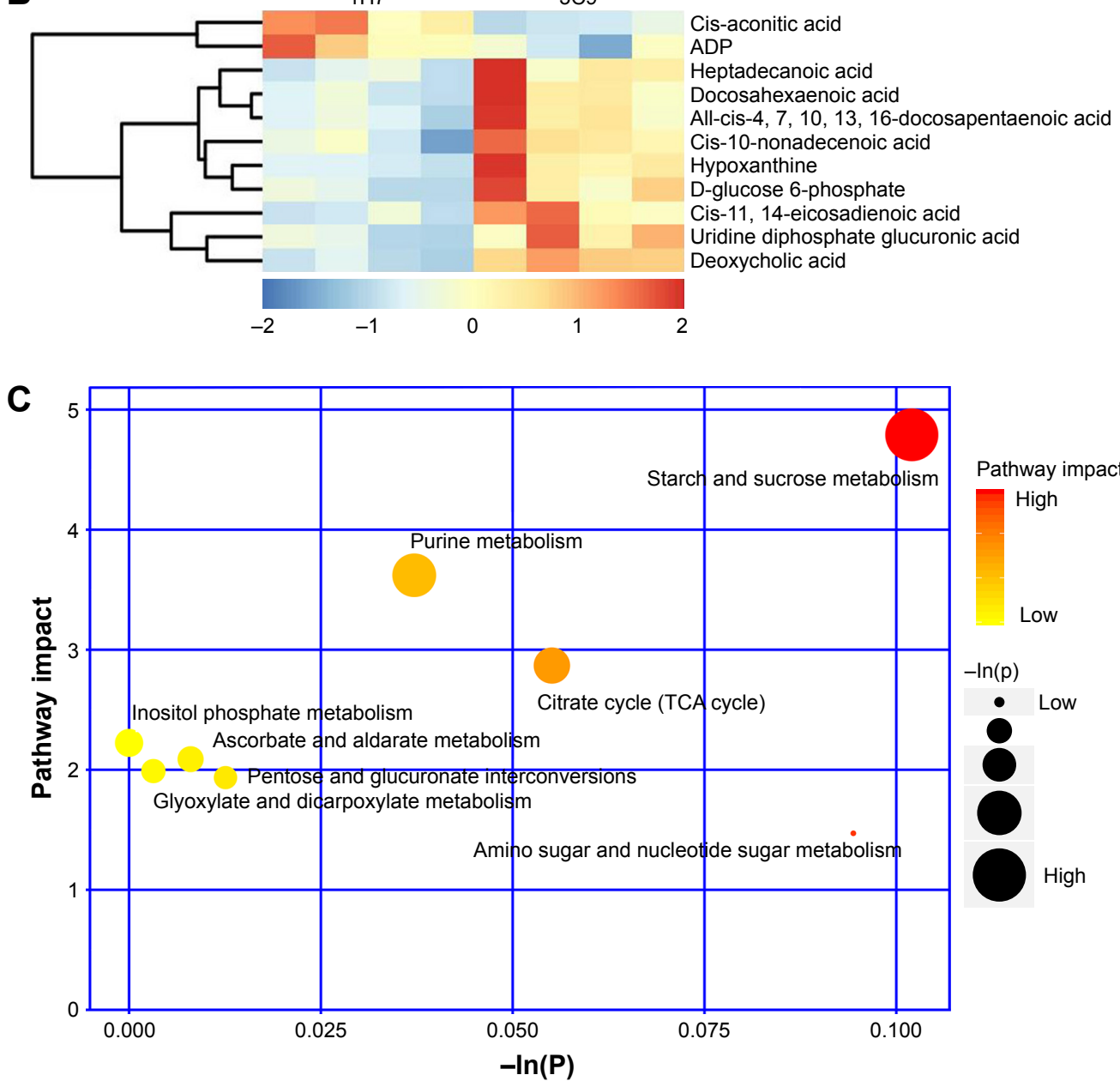

Figure 4 TNF- $\alpha$ knockout affected the metabolism profile of K562.

Notes: (A) Permutation test OPLS-DA model for $3 C 9$ vs $1 \mathrm{H} 7$ group. The green dots represent values of $\mathrm{R}^{2} \mathrm{Y}$ obtained from the permutation test; the square blue dots represent $\mathrm{Q}^{2}$ values obtained from the permutation test; the 2 dashed lines represent the regression lines of $\mathrm{R}^{2} \mathrm{Y}$ and $\mathrm{Q}^{2}$, respectively. (B) Heat map of hierarchical clustering analysis on differential metabolites in $3 \mathrm{C} 9$ and $\mathrm{IH} 7$ cells. (C) Pathway analysis of differential metabolites in $3 \mathrm{C} 9$ and $\mathrm{IH} 7$ cells. The $x$ axis represents the pathway impact, and the $y$ axis represents the pathway enrichment. Larger sizes and darker colors represent increased pathway enrichment and higher pathway impact values, respectively. Abbreviation: OPLS-DA, orthogonal projections to latent structures-discriminant analysis. 


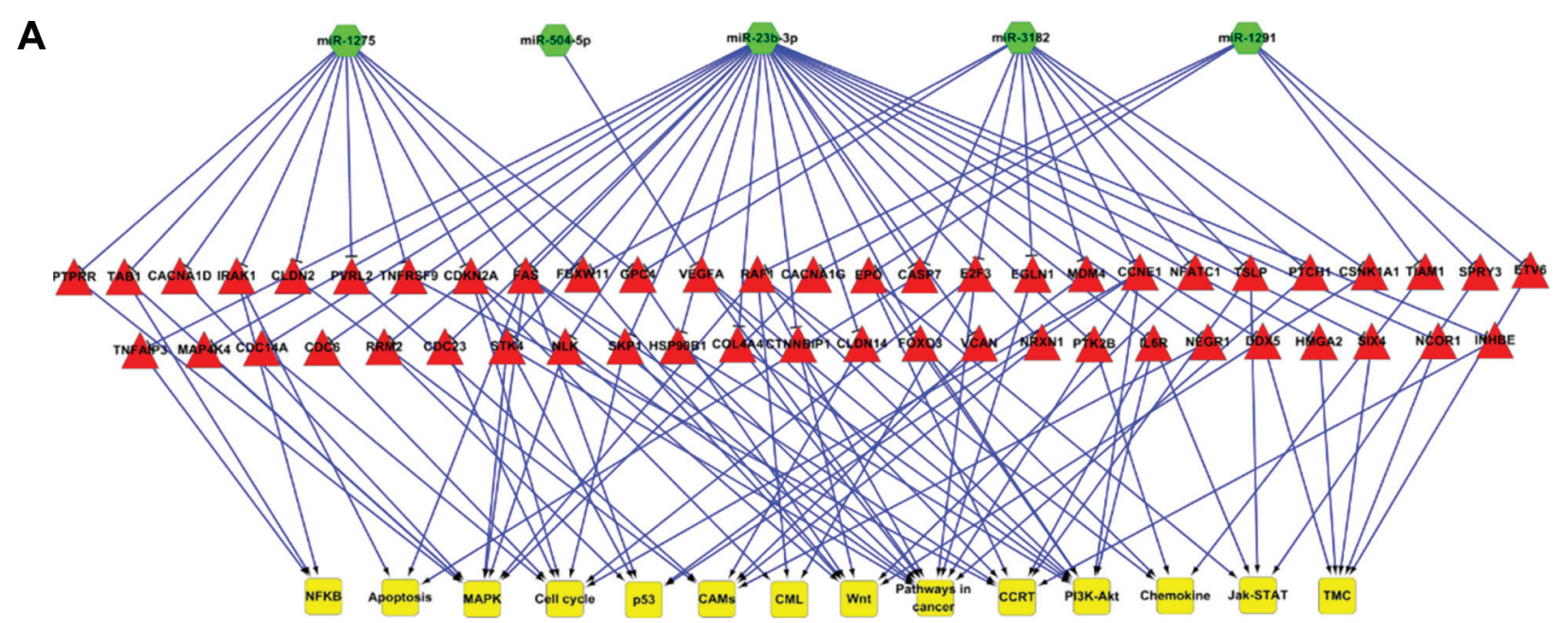

B

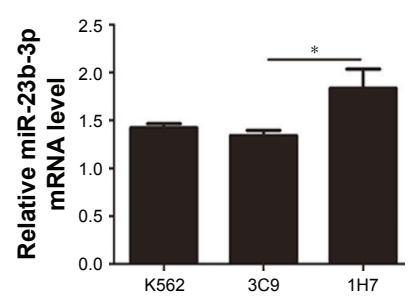

C

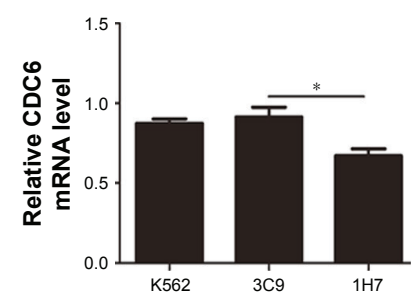

D

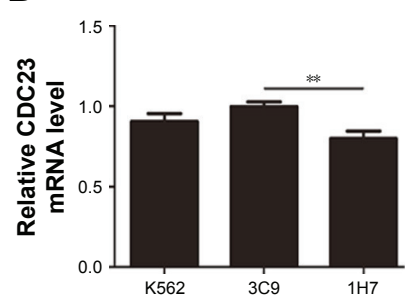

E

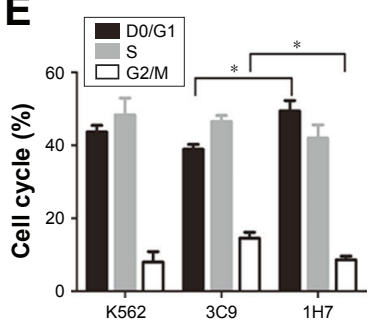

Figure 5 TNF- $\alpha$ knockout affected the expression of miRNAs.

Notes: (A) The regulatory analysis of differentially expressed miRNAs and predicted TFs in cancer-related pathways in wild-type control cells and TNF- $\alpha$ knockout cells. Green, differentially expressed miRNAs; red, target genes regulated by differentially expressed miRNAs; and yellow, pathways related to differentially expressed miRNAs. (B) The relative expression of miR-23b-3p by qRT-PCR. U6 was used as the internal control. (C and D) The relative mRNA expression of CDC6 and CDC23. GAPDH was used as an internal control. (E) Cell cycle analysis of TNF- $\alpha$ knockout cells and control cells. Error bars represent mean \pm SEM. $*_{p}<0.05$ and $* * p<0.01$.

Abbreviation: qRT-PCR, quantitative reverse transcription PCR.

miR-1291, among which the function of miR-23b-3p was the most important. Predicted functional genes regulated by miR-23b-3p include E2F3, CDC6, CCNE1, STK4, FOXD3 and FAS, which were mainly enriched in the cell cycle, CML, P13K-Akt and pathways in cancer (Figure 5A). We verified the small RNA-seq results by RT-PCR and flow cytometry. miR-23b-3p was highly expressed in $1 \mathrm{H} 7$ cells, whereas CDC6 and CDC23 mRNA expression were downregulated, demonstrating that the potential target gene of miR-23b-3p may be CDC6 and CDC23 (Figure 5B-D). Consistent with the above mentioned RNA-seq results, the cell cycle of TNF- $\alpha$ knockout cells was more active than control cells (Figure 5E).

\section{Discussion}

TNF- $\alpha$ is often produced by malignant and immune cells present in the inflammatory tissue surrounding tumors. There are 2 bioactive forms of TNF- $\alpha$ protein as follows: the transmembrane form (tmTNF- $\alpha$ ) and the secretory form $(\mathrm{sTNF}-\alpha) \cdot{ }^{13}$ Numerous publications have reported that TNF- $\alpha$ is overproduced in the peripheral blood or bone marrow of patients with myelodysplastic syndrome, AML and CML. ${ }^{10,18,19}$ Regardless of its source, TNF- $\alpha$ can contribute to tumorigenesis by creating a tumor-supportive inflammatory microenvironment or through direct effects on malignant cells. ${ }^{20}$

CML SPCs produce more TNF- $\alpha$ than their normal counterparts, and inhibition of autocrine TNF- $\alpha$ signaling via a small-molecule inhibitor induces the apoptosis of CML SPCs. When combined with nilotinib, the effect is more significant and can even eliminate primitive quiescent CML LSCs. ${ }^{10}$ tmTNF- $\alpha$ is preferentially expressed by LSCs. Knockdown of tmTNF- $\alpha$ expression renders leukemia cells more sensitive to chemotherapy in vitro. Targeting tmTNF- $\alpha$ by an $\mathrm{mAb}$ resulted in leukemia cell killing in vitro and inhibited leukemia cell growth in vivo while simultaneously sparing normal hematopoietic cells. ${ }^{21}$ Gonzalez-Flores et $\mathrm{al}^{22}$ also found that TNF- $\alpha$ induced apoptosis in human myeloid leukemia cell lines HL-60 and K562, which was dependent on intracellular ROS generation. All these data demonstrate that TNF- $\alpha$ is a preferred choice for targeted therapy. In our research, TNF- $\alpha$ knockout did not influence the apoptosis of K562 cells; however, when incubated with a low concentration of imatinib, the apoptosis rate was significantly increased. This may be because the effect of the single TNF- $\alpha$ gene was compensated by the powerful background of BCR-ABL1 tyrosine kinase activity. When BCR-ABL1 
activity was inhibited by imatinib, the effect of the TNF- $\alpha$ deletion in K562 cells became apparent. The proapoptotic role of TNF- $\alpha$ in K562 cells became less obvious after incubation with imatinib for $48 \mathrm{~h}$; this could be due to the off-target effects of long-term treatment with imatinib.

TNF- $\alpha$ knockout impaired the colony-forming capacity of K562 cells, which is consistent with previous studies. ${ }^{10}$ The combination of nilotinib and TNF- $\alpha$ inhibitor reduced CML CD34+cells' colony forming cell output, including BFU-E/ CFU-E and CFU-GM. ${ }^{10}$ Exogenous TNF promotes AML leukemia cell growth but significantly represses the growth of normal HSPC colonies. ${ }^{23}$ On account of the difficulty in CRISPR/Cas 9-mediated TNF- $\alpha$ knockout in primary CML CD34+cells and ethical restriction, we did not conduct cell experiments on human samples.

Anti-TNF antibody treatment significantly delayed the leukemia development capacity of AML leukemia cells in an in vivo transplantation model. ${ }^{23}$ There were similar results by Zhou et al that tmTNF mAb treatment markedly improved the survival of mice transplanted with AML cells and consistently impaired AML engraftment in bone marrow and liver. ${ }^{21}$ In our transplantation model, TNF knockout delayed tumor formation. At the end of the experiment, the tumor volumes and tumor weights of mice in the control group were higher than those in the TNF- $\alpha$ knockout group. These data consistently demonstrated that TNF- $\alpha$ can promote tumorigenesis in vivo.

On the other hand, an increased percentage of mature NK cells and the TNF- $\alpha /$ IFN- $\gamma$ cytokine secretion by NK cells correlated with sustained remission after discontinuation. ${ }^{24}$ The possible mechanism is that an increased amount of mature NK cells may be directly acting against leukemia cells and potentiating adaptive immune responses against leukemia. In our experiment, TNF- $\alpha$ knockout impaired the proliferation and tumorigenesis of K562 cells. In theory, these phenomena are related to nonrelapse after TKI discontinuation. The reason for the discrepancy may be that llander et $\mathrm{al}^{24}$ studied the whole human immune microenvironment, whereas we researched a single cell line, which lacked communication with other cells.

Cancer pathogenesis has long been linked to metabolic changes required to maintain aberrant survival and growth of malignant cells. ${ }^{25}$ Cellular transformation and oncogenesis are intimately linked to the altered metabolism that drives cancer progression. ${ }^{26}$ Deficiency of TNF receptor-associated factor 3 (TRAF3) increases glucose uptake, anaerobic glycolysis and oxidative phosphorylation in B cells. ${ }^{27}$ $\mathrm{B}$ cell-specific deletion of TRAF3 in mice is associated with enhanced $B$ cell survival and an increased incidence of B cell lymphoma and multiple myeloma. ${ }^{28}$ In our study, TNF- $\alpha$ knockout promoted starch and sucrose metabolism, pentose and glucuronate interconversions and amino sugar and nucleotide sugar metabolism and downregulated the citrate cycle. The differential metabolite heptadecanoic acid was increased, and ADP was decreased, in TNF- $\alpha$ knockout cells. The 2 metabolites were involved in purine metabolism. Fumaric acid, an intermediate product of the citrate cycle, significantly inhibited TNF- $\alpha$-induced eotaxin-1 expression in mouse fibroblasts, which was mediated through the inhibition of p38 MAPK-dependent NF- $\mathrm{KB}$ signaling. ${ }^{29}$ Inhibition of glycolysis with 2-deoxyglucose significantly suppressed lipopolysaccharide-induced interleukin-1 $\beta$ and TNF- $\alpha$ expression in mice. ${ }^{30}$ These data indirectly demonstrate that TNF- $\alpha$ is closely related to glycometabolism.

miRNAs, a class of highly conserved small noncoding RNA, participate in a wide range of biological processes by regulating $\mathrm{mRNA}$ translation at the posttranscriptional level. ${ }^{31}$ Numerous miRNAs promote tumorigenesis and cancer progression by enhancing tumor growth, angiogenesis, invasion and immune evasion, whereas others have tumor suppressive effects. ${ }^{32} \mathrm{Wu}$ et $\mathrm{al}^{33}$ found that TNF- $\alpha$ improves expression of miR-146a-5p, and miR-146-5p inhibits adipogenesis by targeting insulin receptor. In our study, TNF- $\alpha$ may downregulate miR-23b-3p, which targeted cell cyclerelated genes CDC6 and CDC23 resulting in cell cycle arrest.

\section{Conclusion}

We demonstrated that TNF- $\alpha$ knockout impaired proliferation, colony-forming capacity and in vivo tumorigenesis capacity of K562 cells. When combined with TKIs, apoptosis was obviously increased. The underlying mechanism could be associated with metabolic pathways and miRNA regulation. This study indicates that interfering TNF- $\alpha$ with small molecular inhibitors may be a potential approach to eliminate CML-LSCs.

\section{Abbreviations}

CCK-8, cell counting kit-8; CML, chronic myeloid leukemia; TKIs, tyrosine kinase inhibitors; LSCs, leukemia stem cells; TNF- $\alpha$, tumor necrosis factor $\alpha$; LC-MS, liquid chromatography-mass spectroscopy; SPC, stem/progenitor cells; LICs, leukemia-initiating cells; HSCs, hematopoietic stem cells; TNFR1, TNF- $\alpha$ receptor 1; RPM, per million reads; OPLS-DA, orthogonal projections to latent structuresdiscriminant analysis; AML, acute myeloid leukemia; TRAF3, TNF receptor-associated factor 3 .

\section{Acknowledgment}

The work was supported by the National Natural Science Foundation of China (NSFC), grant number 81500136 to Xiaojian Zhu and 81700142 to Qing Li. 


\section{Disclosure}

The authors report no conflicts of interest in this work.

\section{References}

1. Jabbour E, Kantarjian H. Chronic myeloid leukemia: 2016 update on diagnosis, therapy, and monitoring. Am J Hematol. 2016;91:252-265. doi:10.1002/ajh.24275

2. Zhou H, Mak PY, Mu H, et al. Combined inhibition of beta-catenin and $\mathrm{Bcr}-\mathrm{Abl}$ synergistically targets tyrosine kinase inhibitor-resistant blast crisis chronic myeloid leukemia blasts and progenitors in vitro and in vivo. Leukemia. 2017;31:2065-2074. doi:10.1038/leu.2017.87

3. Pfirrmann M, Baccarani M, Saussele S, et al. Prognosis of long-term survival considering disease-specific death in patients with chronic myeloid leukemia. Leukemia. 2016;30:48-56. doi:10.1038/leu.2015.261

4. Apperley JF. Chronic myeloid leukaemia. Lancet. 2015;385:1447-1459. doi:10.1016/S0140-6736(13)62120-0

5. Mahon FX, Rea D, Guilhot J, et al. Discontinuation of imatinib in patients with chronic myeloid leukaemia who have maintained complete molecular remission for at least 2 years: the prospective, multicentre Stop Imatinib (STIM) trial. Lancet Oncol. 2010;11:1029-1035. doi:10.1016/S1470-2045(10)70233-3

6. Kumagai T, Nakaseko C, Nishiwaki K, et al. Dasatinib cessation after deep molecular response exceeding 2 years and natural killer cell transition during dasatinib consolidation. Cancer Sci. 2018;109:182192. doi: $10.1111 /$ cas. 13430

7. Rea D, Nicolini FE, Tulliez M, et al. Discontinuation of dasatinib or nilotinib in chronic myeloid leukemia: interim analysis of the STOP 2G-TKI study. Blood. 2017;129:846-854. doi:10.1182/blood-2016-09-742205

8. Holyoake TL, Vetrie D. The chronic myeloid leukemia stem cell: stemming the tide of persistence. Blood. 2017;129:1595-1606. doi:10.1182/ blood-2016-09-696013

9. Jaco I, Annibaldi A, Lalaoui N, et al. MK2 phosphorylates RIPK1 to prevent TNF-induced cell death. Mol Cell. 2017;66:698-710.e5. doi:10.1016/j.molcel.2017.05.003

10. Gallipoli P, Pellicano F, Morrison H, et al. Autocrine TNF-alpha production supports CML stem and progenitor cell survival and enhances their proliferation. Blood. 2013;122:3335-3339. doi:10.1182/blood2013-02-485607

11. Kagoya Y, Yoshimi A, Kataoka K, et al. Positive feedback between NF-kappaB and TNF-alpha promotes leukemia-initiating cell capacity. J Clin Invest. 2014;124:528-542. doi:10.1172/JCI68101

12. Pronk CJ, Veiby OP, Bryder D, et al. Tumor necrosis factor restricts hematopoietic stem cell activity in mice: involvement of two distinct receptors. J Exp Med. 2011;208:1563-1570. doi:10.1084/jem. 20110752

13. Zhou X, Li Z, Zhou J. Tumor necrosis factor alpha in the onset and progression of leukemia. Exp Hematol. 2017;45:17-26. doi:10.1016/j. exphem.2016.10.005

14. Ito K, Ito K. Metabolism and the control of cell fate decisions and stem cell renewal. Annu Rev Cell Dev Biol. 2016;32:399-409. doi:10.1146/ annurev-cellbio-111315-125134

15. Borriello A, Caldarelli I, Bencivenga D, et al. Tyrosine kinase inhibitors and mesenchymal stromal cells: effects on self-renewal, commitment and functions. Oncotarget. 2017;8:5540-5565. doi:10.18632/ oncotarget.12649

OncoTargets and Therapy

\section{Publish your work in this journal}

OncoTargets and Therapy is an international, peer-reviewed, open access journal focusing on the pathological basis of all cancers, potential targets for therapy and treatment protocols employed to improve the management of cancer patients. The journal also focuses on the impact of management programs and new therapeutic agents and protocols on
16. Wang YH, Israelsen WJ, Lee D, et al. Cell-state-specific metabolic dependency in hematopoiesis and leukemogenesis. Cell. 2014;158: 1309-1323. doi:10.1016/j.cell.2014.07.048

17. Kuntz EM, Baquero P, Michie AM, et al. Targeting mitochondrial oxidative phosphorylation eradicates therapy-resistant chronic myeloid leukemia stem cells. Nat Med. 2017;23:1234-1240. doi:10.1038/nm.4399

18. Wang M, Zhang C, Tian T, et al. Increased regulatory T cells in peripheral blood of acute myeloid leukemia patients rely on Tumor Necrosis Factor (TNF)-alpha-TNF receptor-2 pathway. Front Immunol. 2018;9: 1274. doi:10.3389/fimmu.2018.01274

19. Lambert $\mathrm{C}, \mathrm{Wu} \mathrm{Y}$, Aanei C. Bone marrow immunity and myelodysplasia. Front Oncol. 2016;6:172. doi:10.3389/fonc.2016.00172

20. Candido J, Hagemann T. Cancer-related inflammation. J Clin Immunol. 2013;33(Suppl 1):S79-S84. doi:10.1007/s10875-012-9847-0

21. Zhou X, Zhou S, Li B, et al. Transmembrane TNF-alpha preferentially expressed by leukemia stem cells and blasts is a potent target for antibody therapy. Blood. 2015;126:1433-1442. doi:10.1182/ blood-2015-01-624833

22. Gonzalez-Flores D, Rodriguez AB, Pariente JA. TNFalpha-induced apoptosis in human myeloid cell lines HL-60 and K562 is dependent of intracellular ROS generation. Mol Cell Biochem. 2014;390:281-287. doi:10.1007/s11010-014-1979-5

23. Volk A, Li J, Xin J, et al. Co-inhibition of NF-kappaB and JNK is synergistic in TNF-expressing human AML. J Exp Med. 2014;211: 1093-1108. doi:10.1084/jem.20130990

24. Ilander M, Olsson-Stromberg U, Schlums H, et al. Increased proportion of mature NK cells is associated with successful imatinib discontinuation in chronic myeloid leukemia. Leukemia. 2017;31:1108-1116. doi:10.1038/leu.2016.360

25. Boroughs LK, DeBerardinis RJ. Metabolic pathways promoting cancer cell survival and growth. Nat Cell Biol.2015;17:351-359. doi:10.1038/ ncb3124

26. Jones RG, Thompson CB. Tumor suppressors and cell metabolism: a recipe for cancer growth. Genes Dev. 2009;23:537-548. doi:10.1101/ gad.1756509

27. Moore CR, Liu Y, Shao C, et al. Specific deletion of TRAF3 in B lymphocytes leads to B-lymphoma development in mice. Leukemia. 2012;26:1122-1127. doi:10.1038/leu.2011.309

28. San MJ. Introduction to a series of reviews on multiple myeloma. Blood. 2015;125:3039-3040. doi:10.1182/blood-2015-01-613596

29. Roh KB, Jung E, Park D, et al. Fumaric acid attenuates the eotaxin-1 expression in TNF-alpha-stimulated fibroblasts by suppressing p38 MAPK-dependent NF-kappaB signaling. Food Chem Toxicol. 2013;58: 423-431. doi:10.1016/j.fct.2013.05.020

30. Tannahill GM, Curtis AM, Adamik J, et al. Succinate is an inflammatory signal that induces IL-1 beta through HIF-1alpha. Nature. 2013;496: 238-242. doi:10.1038/nature11986

31. Neudecker V, Brodsky KS, Kreth S, et al. Emerging roles for MicroRNAs in perioperative medicine. Anesthesiology. 2016;124:489-506. doi:10.1097/ALN.0000000000000969

32. Yeh $\mathrm{CH}$, Moles R, Nicot C. Clinical significance of microRNAs in chronic and acute human leukemia. Mol Cancer. 2016;15:37. doi:10.1186/s12943-016-0518-2

33. Wu D, Xi QY, Cheng X, et al. miR-146a-5p inhibits TNF-alphainduced adipogenesis via targeting insulin receptor in primary porcine adipocytes. J Lipid Res. 2016;57:1360-1372. doi:10.1194/j1r.M062497

\section{Dovepress}

patient perspectives such as quality of life, adherence and satisfaction The manuscript management system is completely online and includes a very quick and fair peer-review system, which is all easy to use. Visit http://www.dovepress.com/testimonials.php to read real quotes from published authors. 